\title{
A Dynamic Bayesian Network Approach to Behavioral Modelling of Elderly People during a Home-based Augmented Reality Balance Physiotherapy Programme
}

\author{
cited as: Georga EI, Gatsios D, Tsakanikas V, Kourou KD, Liston M, Pavlou M, Kikidis D, \\ Bibas A, Nikitas C, Bamiou DE, Fotiadis DI. A Dynamic Bayesian Network Approach to \\ Behavioral Modelling of Elderly People during a Home-based Augmented Reality Balance \\ Physiotherapy Programme. Annu Int Conf IEEE Eng Med Biol Soc. 2020 Jul;2020:5544- \\ 5547. doi: 10.1109/EMBC44109.2020.9175435. PMID: 33019234.
}

\begin{abstract}
Eleni I. Georga, Dimitrios Gatsios, Vasilis Tsakanikas, Konstantina D. Kourou, Matthew Liston, Marousa Pavlou, Dimitrios Kikidis, Athanasios Bibas, Christos Nikitas, Doris Eva Bamiou, and Dimitrios I. Fotiadis, Fellow Member, IEEE
\end{abstract}

\begin{abstract}
In this study, we propose a dynamic Bayesian network (DBN)-based approach to behavioral modelling of community dwelling older adults at risk for falls during the daily sessions of a hologram-enabled vestibular rehabilitation therapy programme. The component of human behavior being modelled is the level of frustration experienced by the user at each exercise, as it is assessed by the NASA Task Load Index. Herein, we present the topology of the DBN and test its inference performance on real-patient data.
\end{abstract}

Clinical Relevance- Precise behavioral modelling will provide an indicator for tailoring the rehabilitation programme to each individual's personal psychological needs.

\section{INTRODUCTION}

More than $1 / 3$ of the population over 40 years old faces some kind of vestibular disorder leading, in most cases, to dizziness and imbalance [1]. Benign paroxysmal positional vertigo (BPPV) is the most commonly reported vestibular disorder accounted for approximately half of cases [2]. Vestibular Rehabilitation Therapy (VRT) has been shown to be very effective for persons suffering from dizziness and balance impairments; VRT decreases duration and intensity of symptoms (e.g. dizziness and postural instability) and lessens the number of falls and fear of falling [3-5]. During supervised, home-based VRT, behavioral outcomes, such as enjoyment and adherence, are associated with interpersonal behaviors psychological needs (i.e. satisfaction and frustration) [6].

According to the NASA Task Load Index (TLX), the subjective workload one individual perceives during his/her interaction with various human-machine interface systems can be defined as a combination of: (i) the mental demand, (ii) the physical demand, (iii) the temporal demand, (iv) the performance, $(\mathrm{v})$ the effort, and (v) the frustration $[7,8]$. In

E. I. G., D. G., V. T., K. D. K. and D. I. F are with the Unit of Medical Technology and Intelligent Information Systems, Department of Materials Science and Engineering, University of Ioannina, Ioannina, GR45110, Greece (corresponding author phone: +302651009006; fax: +302651008889; e-mail: fotiadis@uoi.gr)

D. I. F is with the Institute of Molecular Biology and Biotechnology, Biomedical Research Department, FORTH, Ioannina, GR 45110 Greece. particular, the frustration level is assessed through the question "How insecure, discouraged, irritated, stressed and annoyed versus secure, gratified, content, relaxed and complacent did you feel during the task?". The frustration scale in the NASATLX focuses on the psychological impact of a task on the subject, and, specifically, frustration, was significantly correlated with the overall workload. Equally important, frustration was shown to provide useful information in distinguishing among types of activities.

In the HOLOBALANCE study [9], the estimation of the frustration level time course one individual experiences during a balance rehabilitation exercise session is treated in the context of dynamic Bayesian networks (DBNs). A number of literature studies adopt DBNs for representing and modelling sequential temporal data and processes, among which human behavior inference [10-14]. DBNs may represent a dynamic stochastic process using a probabilistic directed graphical model (i) considering the degree of uncertainty in the examined dynamical system, (ii) allowing reasoning about both spatial and temporal dynamics in a system, and (iii) combining knowledge elicited by the experts and a parameterized (data-driven) solution.

\section{MATERIALS AND METHODS}

\section{A. Theoretical Formulation of Behavioral Modelling as a DBN Learning Problem}

As it is shown in Fig. 1, we consider that each exercise session encompasses $T$ discrete tasks $\operatorname{Task}_{i}$, with $i=1, \ldots, T$, which define accordingly an equal number of time points $t_{i}$. The identification of one individual's frustration level during each task can be formulated as a sequence learning problem. Let us denote $U^{(t)} \in R^{d}$ and $Y^{(t)}$ the vector of input variables (i.e. an individual's context) and the output variable (i.e. frustration level), respectively, pertaining to the task

M. L., and M. P. are with the Centre for Human and Applied Physiological Sciences, King's College London, London, United Kingdom (email: Marousa.Pavlou@kcl.ac.uk).

D. K., A. B., and C. N. are with First Department of Otolaryngology-Head and Neck Surgery, 'Hippokrateio' General Hospital, National Kapodistrian University of Athens, Athens, Greece (email: dimitriskikidis@yahoo.com).

D. E. B. is with the UCL Ear Institute \& University College London Hospitals Biomedical Research Centre Hearing \& Deafness, London, United Kingdom (e-mail: d.bamiou@ucl.ac.uk). 
performed at time $t$. Assuming that one individual's frustration during a task is affected by the current observations $U^{(t)}$ and historical states $Z^{(t-k \Delta t)}$ of the underlying system (i.e. behaviour of an individual during a set of consecutive tasks), with $k \Delta t$ denoting the history length and $k>0$, the posterior

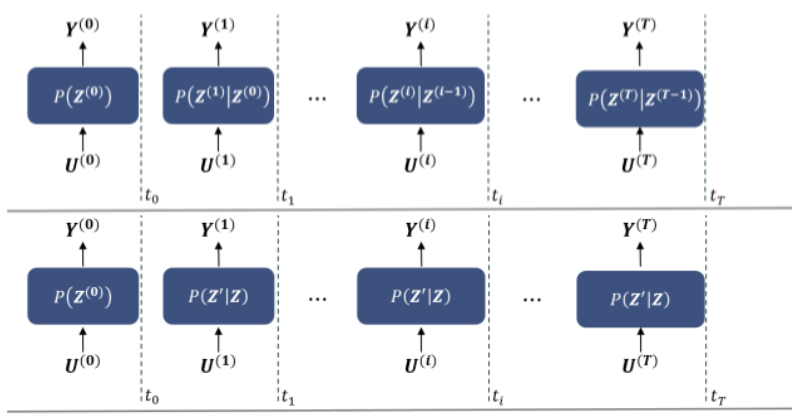

Figure 1. Schematic representation of the mapping of an exercise session input/output variables to DBNs graphical notation.

probability $P\left(Y^{(t)} \mid U^{(t)}, Z^{(t-k \Delta t)}\right)$ can be estimated by employing inference and parameter/structure learning algorithms of the DBN class.

DBNs represent and estimate probability distributions over trajectories, $P\left(Z^{(0)}, Z^{(1)}, \ldots, Z^{(T)}\right)$, i.e. a system that is dynamically changing or evolving over time [15]. The state of the system at time $t$ is represented by a set of random variables:

$$
Z^{(t)}=\left\{U^{(t)}, X^{(t)}, Y^{(t)}\right\}, \mathrm{t}=0, \ldots, \mathrm{T},
$$

where $U^{(t)}, X^{(t)}$, and $Y^{(t)}$ represent the input, hidden and output variables of the model, respectively. A DBN is defined as a pair $\left(B_{1}, B_{\rightarrow}\right)$, where $B_{1}$ is a Bayesian network associated with an initial state distribution of the state variables $P\left(Z^{(0)}\right)$ and $B_{\rightarrow}$ is a two-slice temporal Bayes net $(2 \mathrm{TBN})$ which defines the transition model $P\left(Z^{(t)} \mid Z^{(t-1)}\right.$ ) (a first order Markov model). $B_{1}$ and $B_{\rightarrow}$, which convey the stochastic dynamics of the examined system, are defined by:

$$
\begin{gathered}
P\left(Z^{(0)}\right), Z^{(0)}=\left\{Z_{1}^{(0)}, Z_{2}^{(0)}, \ldots, Z_{d}^{(0)}\right\}, \\
P\left(Z^{(t)} \mid Z^{(t-1)}\right)=\prod_{i=1}^{N} P\left(Z_{i}^{(t)} \mid P a\left(Z_{i}^{(t)}\right)\right),
\end{gathered}
$$

where $Z_{i}^{(t)}$ is the $i^{\text {th }}$ random variable (or node) at time $t$, and $\operatorname{Pa}\left(Z_{i}^{(t)}\right)$ are the parental nodes of $Z_{i}^{(t)}$ in the current or previous slice. DBNs obey the Markov assumption and the assumption of time invariance. Thus, by unfolding the 2TBN in time, the joint probability distribution for a sequence of length $T$ is given by:

$$
P\left(Z_{1: T}\right)=\prod_{t=1}^{T} \prod_{i=1}^{d} P\left(Z_{i}^{(t)} \mid \operatorname{Pa}\left(Z_{i}^{(t)}\right)\right) .
$$

\section{B. Methodology of DBN Model Development}

The development of the HOLOBALANCE DBN entails: (i) the specification of the state space $Z^{(t)}$, (ii) the specification of the structure of the DBN (i.e. inter-time-slice, and intratime-slice edges), (iii) the initialization of the state distribution $P\left(Z^{(0)}\right)$ and the conditional probability distributions $P\left(Z^{(t)} \mid Z^{(t-1)}\right)$ (Model $M_{0}$ ), (iv) Expectation-Maximization (EM) learning of the model's parameters (Model $M_{E M}$ ), (v) the inference of the probability of frustration given the evidence (e) of observed dynamic or static data $P$ (Frustration $\mid e$ ), and (vi) the evaluation of the performance of $M_{E M}$ using welldefined classification metrics.

\section{A. Model Specification}

\begin{tabular}{|c|c|c|}
\hline \multirow{5}{*}{ 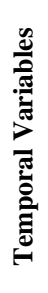 } & Symptoms & $\begin{array}{l}\text { Headache symptoms scale; Dizziness rating } \\
\text { scale; Disorientation; Blurry/jumpy vision }\end{array}$ \\
\hline & Stress Level & Stress level before / during /after the task \\
\hline & Prompts & Re-instructions \\
\hline & $\begin{array}{l}\text { Qualitative } \\
\text { Task Data }\end{array}$ & $\begin{array}{l}\text { Mental demand; Physical and temporal } \\
\text { demand; Performance score; Adherence to } \\
\text { instructions }\end{array}$ \\
\hline & $\begin{array}{l}\text { Treatment } \\
\text { Variables }\end{array}$ & $\begin{array}{l}\text { The weekly program with the tasks/exercises } \\
\text { to be performed daily }\end{array}$ \\
\hline \multirow{3}{*}{ 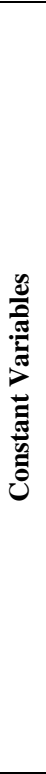 } & $\begin{array}{l}\text { Personal } \\
\text { Characte- } \\
\text { ristics }\end{array}$ & $\begin{array}{l}\text { Socio-demographic information: Year of } \\
\text { birth, gender, education, occupation, marital } \\
\text { status, experience with technology, other; } \\
\text { Social support: Significant others, carers; } \\
\text { Adherence history: How compliant the } \\
\text { patient was in previous rehabilitation plans }\end{array}$ \\
\hline & $\begin{array}{l}\text { Medical } \\
\text { Condition } \\
\text { Outcome } \\
\text { Measures }\end{array}$ & $\begin{array}{l}\text { The Montreal Cognitive Assessment } \\
\text { (MOCA); The Rapid Assessment of Physical } \\
\text { Activity (RAPA); The World Health } \\
\text { Organisation Disability Assessment } \\
\text { Schedule } 2 \text { (WHODAS 2.0); The Cambridge } \\
\text { Neuropsychological Test Automated Battery } \\
\text { (CANTAB); The Activities Specific Balance } \\
\text { Confidence Scale (ABC-Scale); The Falls } \\
\text { Efficacy Scale - International (FES-I); The } \\
\text { EQ5D The Environmental Mobility Scale } \\
\text { (EMS); The Mini BESTest Functional Gait } \\
\text { Assessment (FGA); The Dizziness Handicap } \\
\text { Inventory (DHI) }\end{array}$ \\
\hline & Symptoms & $\begin{array}{l}\text { Vertigo, dizziness, nausea, imbalance, falls, } \\
\text { difficulty walking in darkness, motion } \\
\text { sickness, visual vertigo, lightheadness, } \\
\text { drunken feeling, deviation during walking, } \\
\text { disorientation, cervicalgia, Tumarkin attacks }\end{array}$ \\
\hline
\end{tabular}

Fig. 2 depicts the current definition of the HOLOBALANCE DBN structure; the state variables $Z^{(t)}=$

TABLE I. The DatasPace IN HOLOBALANCE StUdy

$\left\{U^{(t)}, X^{(t)}, Y^{(t)}\right\}$ and the cross-sectional (intra-slice) as well as temporal (inter-slice) connectivity among them. Frustration', which is defined as a 3-class variable (i.e. Low, Moderate, High), constitutes the output $Y^{(t)}$ of the DBN, whereas the input $U^{(t)}$ is drawn on the baseline (static variables) and observed (dynamic) data shown in Table I [16]. To reduce complexity, the 'Frustration', 'Symptoms' and 'Performance' are defined as Leaky Noisy-MAX nodes. Conforming with the Markov assumption, the order $k$ of the identified temporal arcs is equal to 1 . We have identified 3 temporal arcs capturing the persistent effect of 'Symptoms', 'Frustration' and 'Stress' as it is transferred from time slice $t$ to time slice $t+1$. For notational simplicity, we assume each sequence is of the same length, the sequence length $T$ of the process is set to 5 .

\section{B. Model Initialization and Learning}

The state distribution $P\left(Z^{(0)}\right)$ and the conditional probability tables (CPTs) expressing $P\left(Z^{(1)} \mid Z^{(0)}\right)$ (Model $M_{0}$ ) were initialized using expert knowledge. Learning algorithms of the topology of the DBN or its parameters will be methodically specified once the HOLOBALANCE dataset becomes available. Model selection depends on the observability of the state variables $Z^{(t)}$ (full observability or 
partial observability in the existence of missing values or hidden variables) as well as on the prior knowledge of the complete topology of the DBN graph.

\section{Model Evaluation}

Let us denote with $P_{p}$, the $p^{\text {th }}$ patient participating in the HOLOBALANCE study. Each exercise session generates a dynamic variables during the last $T$ time points of the exercise session for 2 indicative subjects, where we observed a considerable variation of the frustration level during the respective exercises. We can observe that the estimated marginal probability $P$ (Frustration) time course correlates well with the true level of 'Frustration', which signifies the

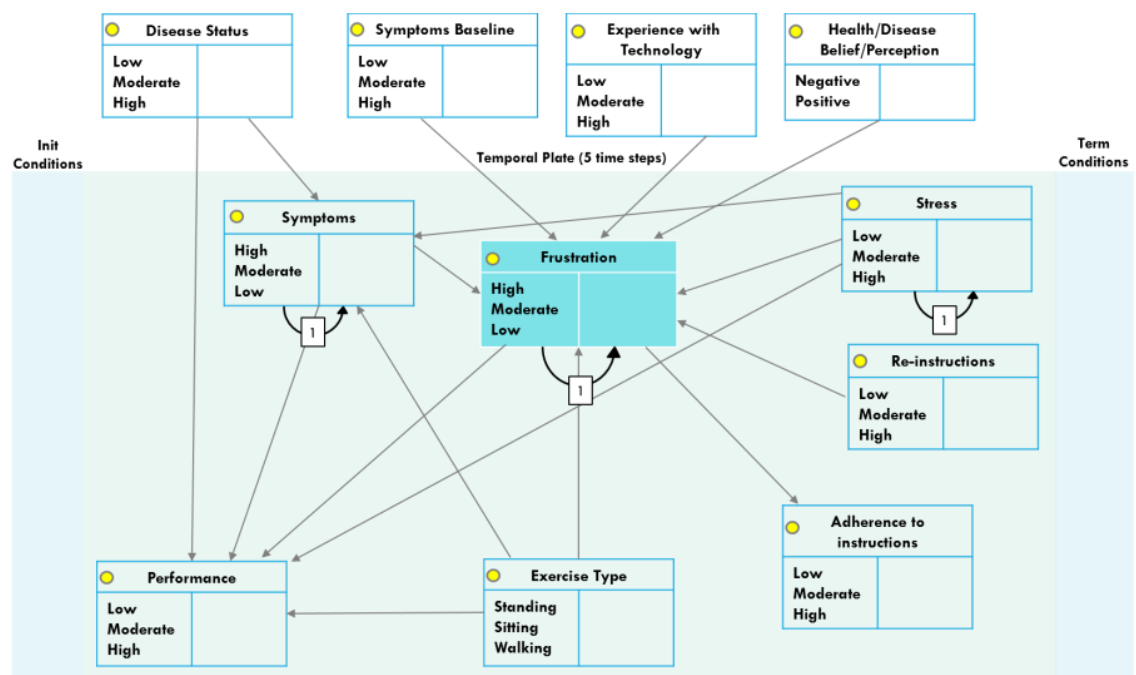

Figure 2. HOLOBALANCE DBN specification.

sequence of values, with $V_{i, j, p}^{(t)}$ denoting the value of variable $V_{i}$ observed at time $t(t=1, \ldots, T)$ on $\operatorname{Day}_{j}(j=1, \ldots, N)$ for patient $P_{p}$. We recall that $V \in Z=\{U, X, Y\}$. A separate dataset $D^{p}=\left\{V_{i, j}^{(t)} \mid t=1, \ldots T, i=1, \ldots d, j=1, \ldots, N\right\}$ is constructed for each subject containing the data sequences pertaining to daily exercise sessions. DBN model training and evaluation can be performed: (i) individually such that $D^{p}=$ $\left\{s_{j} \mid j=1, \ldots, N\right\}=\left\{V_{i}^{(t)} \mid t=1, \ldots T, i=1, \ldots d,\right\}$, and (ii) globally by concatenating $D^{p}$ for $p=1, \ldots, P$, such that $D=$ $\left\{V_{i, j, p}^{(t)} \mid t=1, \ldots T, i=1, \ldots d, j=1, \ldots, N, p=1, \ldots, P\right\} . \mathrm{A}$ repeated sampling scheme will be employed to build and estimate the classification performance of the DBN model, considering internal tuning of model's hyper-parameter. Model evaluation will be based on well-defined performance measures for multi-class problems [17].

\section{Inference}

Inference of the P(Frustration $\mid e$ ), where $e$ denotes the evidence of observed dynamic or static data, constitutes a filtering problem; the current belief state is computed given all evidence from the past $P\left(X_{t} \mid y_{1: t}\right)$. The Clustering algorithm has been currently evaluated for exact inference [16]. Nonetheless, considering exact inference is computationally intensive in discrete-state models, approximate deterministic or stochastic inference algorithms will be examined as well.

\section{RESULTS}

Currently, we have tested the inference performance of the DBN model $M_{0}$ on 5 real data trajectories. Each patient performed up to 36 exercises, which are all the flowchart exercises with progressions, using the HOLOBALANCE sensing module, whereas 'Frustration' was assessed after exercise was performed. Fig. 3 depicts the time series of the specified topology of the DBN and the associated CPTs of $M_{0}$ can explain the behavioural dynamics at each exercise.

\section{DISCUSSION AND CONCLUSIONS}

Behavioral modelling, as it is captured through the estimation of the user's frustration level at each task of an exercise session, has been addressed by a DBN of both static and dynamic data and their cross-sectional and temporal interdependencies. The structure and the parameters have been specified based solely on knowledge provided by the clinicians, and the resulting DBN was found to produce sufficiently accurate estimates of the true frustration level of the 5 participants. However, learning primarily the parameters of the DBN using each patient's data trajectories, obtained from the HOLOBALANCE study, will reveal the data driven CPDs, whereas structure learning will reveal the strength of the defined connections (graph topology). Therefore, the DBN data modelling pipeline, organized either in an individualized or global learning mode, will incorporate (i) parameters learning assuming the currently-defined structure, and (ii) both parameters and structure learning aiming at validating existing edges or mining new ones. Moreover, the DBN model's input space shall be refined during the model training, selection and validation phases, especially as regards the effect of the type of exercises on the observed variables. An accurate model of the frustration, which is experienced during a task by a specific user or a group of users, could support physicians in designing more effective balance rehabilitation schemes through the explainability or interpretability of DBN's output per se.

\section{ACKNOWLEDGMENT}

This work has received funding from the European Union's Horizon 2020 research and innovation programme under grant agreement No 769574. 


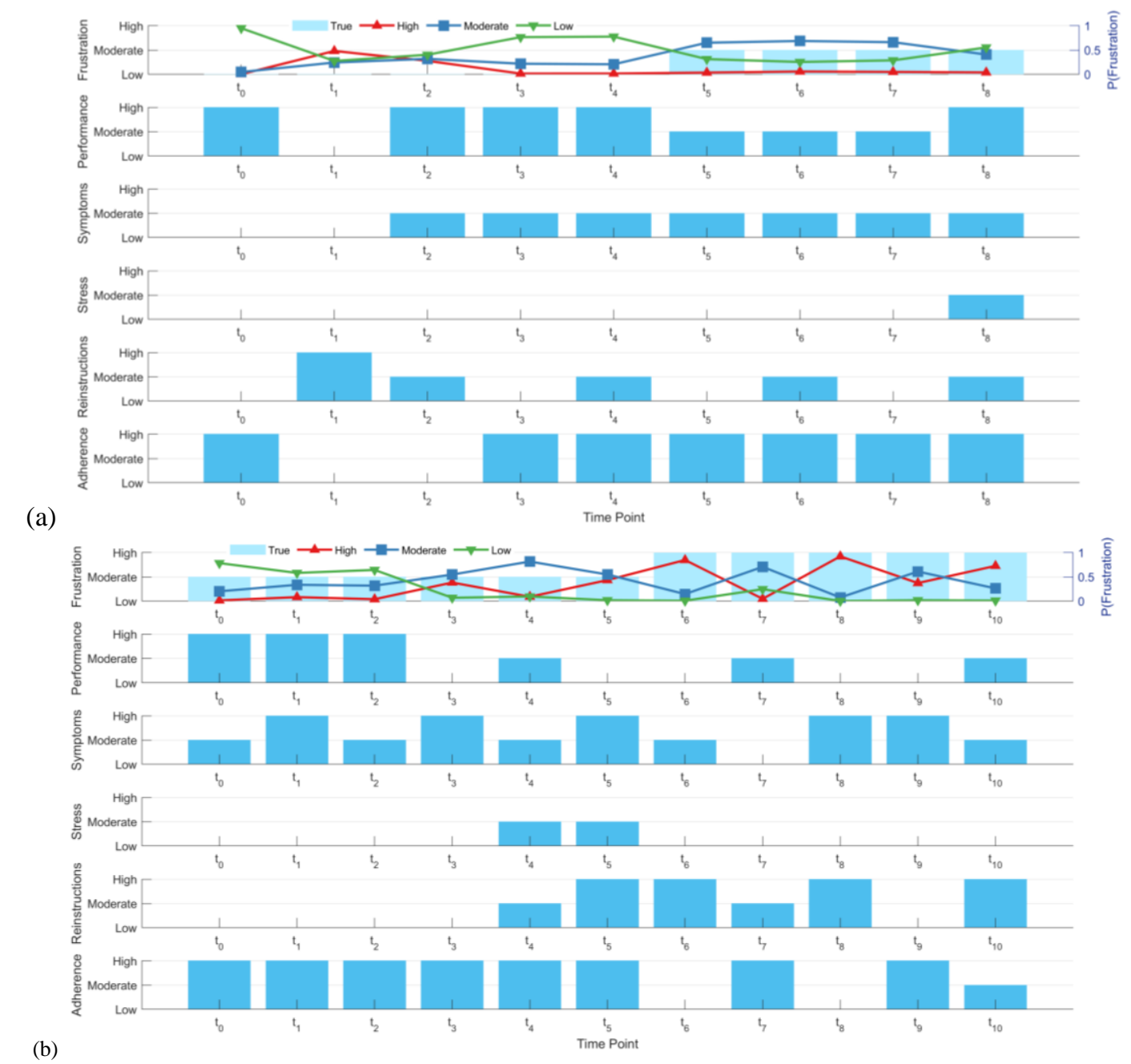

Figure 3. Exact inference of the frustration level of (a) Subject 1 and (b) Subject 5 based on the DBN $M_{0}$. Right y-axis of the top plot specifies the marginal probability $P$ (Frustration), where red, blue and green lines indicate the estimated conditional probabilities $P(($ Frustration $=$ High $) \mid e)$, $(($ Frustration $=$ Moderate $) \mid e)$ and $P(($ Frustration $=$ Low $) \mid e)$. Left y-axis of each plot specifies the observed level of each variable.

\section{REFERENCES}

[1] Y. Agrawal, J. P. Carey, C. C. Della Santina, M. C. Schubert, and L. B. Minor, "Disorders of balance and vestibular function in US adults: data from the National Health and Nutrition Examination Survey, 20012004," Arch Intern Med, vol. 169, pp. 938-44, May 252009.

[2] M. Von Brevern, A. Radtke, F. Lezius, M. Feldmann, T. Ziese, T. Lempert, et al., "Epidemiology of benign paroxysmal positional vertigo: a population based study," Journal of Neurology, Neurosurgery \& Psychiatry, vol. 78, pp. 710-715, 2007.

[3] P. M. Dunlap, J. M. Holmberg, and S. L. Whitney, "Vestibular rehabilitation: advances in peripheral and central vestibular disorders," Current opinion in neurology, vol. 32, pp. 137-144, 2019.

[4] S. L. Hillier and M. McDonnell, "Vestibular rehabilitation for unilateral peripheral vestibular dysfunction," Cochrane database of systematic reviews, 2007.

[5] S. J. Herdman, M. C. Schubert, and R. J. Tusa, "Strategies for balance rehabilitation: fall risk and treatment," Annals of the New York Academy of Sciences, vol. 942, pp. 394-412, 2001.

[6] F. Rodrigues, T. Bento, L. Cid, H. Pereira Neiva, D. Teixeira, J. Moutao, et al., "Can Interpersonal Behavior Influence the Persistence and Adherence to Physical Exercise Practice in Adults? A Systematic Review," Front Psychol, vol. 9, p. 2141, 2018.

[7] S. G. Hart and L. E. Staveland, "Development of NASA-TLX (Task Load Index): Results of Empirical and Theoretical Research," in
Advances in Psychology. vol. 52, P. A. Hancock and N. Meshkati, Eds., ed: North-Holland, 1988, pp. 139-183.

[8] S. G. Hart, "Nasa-Task Load Index (NASA-TLX); 20 Years Later," Proceedings of the Human Factors and Ergonomics Society Annual Meeting, vol. 50, pp. 904-908, 2006/10/01 2006.

[9] D. Gatsios, D. E. Bamiou, S. Costafreda, E. I. Georga, K. K. Kourou, T. Exarchos, et al., "Empowering Community Dwelling Older Citizens to Improve Their Balance with a Novel Technology Platform," Cham, 2020, pp. 983-988.

[10] N. Khakzad, G. Landucci, and G. Reniers, "Application of dynamic Bayesian network to performance assessment of fire protection systems during domino effects," Reliability Engineering \& System Safety, vol. 167, pp. 232-247, 2017/11/01/2017.

[11] J. J. Dabrowski and J. P. De Villiers, "A unified model for contextbased behavioural modelling and classification," Expert Systems with Applications, vol. 42, pp. 6738-6757, 2015.

[12] M. Patel, J. Valls Miro, and G. Dissanayake, "Dynamic Bayesian Networks for Learning Interactions between Assistive Robotic Walker and Human Users," Berlin, Heidelberg, 2010, pp. 333-340.

[13] K. A. Tahboub, "Intelligent Human-Machine Interaction Based on Dynamic Bayesian Networks Probabilistic Intention Recognition," Journal of Intelligent and Robotic Systems, vol. 45, pp. 31-52, 2006/01/01 2006.

[14] T.-Y. Ma, "Bayesian Networks for Multimodal Mode Choice Behavior Modelling: A Case Study for the Cross Border Workers of 
Luxembourg," Transportation Research Procedia, vol. 10, pp. 870880, 2015/01/01/ 2015.

[15] K. P. Murphy, "Dynamic bayesian networks: representation, inference and learning," University of California, Berkeley, 2002.

[16] M. J. Druzdzel, "SMILE: Structural Modeling, Inference, and Learning Engine and GeNIe: a development environment for graphical decisiontheoretic models," presented at the Proceedings of the sixteenth national conference on Artificial intelligence and the eleventh Innovative applications of artificial intelligence conference innovative applications of artificial intelligence, Orlando, Florida, USA, 1999.

[17] M. Sokolova and G. Lapalme, "A systematic analysis of performance measures for classification tasks," Information Processing \& Management, vol. 45, pp. 427-437, 2009/07/01/ 2009. 


\section{COPYRIGHT NOTICE}

IEEE Catalog Number: CFP20EMB-ART

ISBN: 978-1-7281-1990-8

Online ISSN: 1558-4615

The 2020 42nd Annual International Conference of the IEEE Engineering in Medicine and Biology Society proceedings was produced for IEEE EMBS by The Printing House.

Copyright and Reprint Permission: Abstracting is permitted with credit to the source. Libraries are permitted to photocopy beyond the limit of U.S. copyright law for private use of patrons those articles in this volume that carry a code at the bottom of the first page, provided the per-copy fee indicated in the code is paid through Copyright Clearance Center, 222 Rosewood Drive, Danvers, MA 01923. For reprint or republication permission, email to IEEE Copyrights Manager at pubs-permissions@ieee.org. All rights reserved. Copyright (C2020 by IEEE.

Copying this product's instructions and/or designs for use on future digital products is prohibited without written permission from The Printing House. The Printing House or its suppliers are not liable for any direct, indirect, special, incidental or consequential damages to your hardware or other software arising out of the use-or the inability to usethe material on this product. This includes, but is not limited to, the loss of data or loss of profit. Adobe, Acrobat and the Acrobat logo are trademarks of Adobe Systems Incorporated or its subsidiaries and may be registered in certain jurisdictions.

If you have questions, please contact:

The Printing House

Phone: +1-608-873-4500

Hours: Monday through Friday, 8 am - 5 pm CST

E-mail: graphics@printinghouseinc.com 